\title{
Validación de criterio y constructo para la creación de un cuestionario de exposición a ruido
}

\author{
Validation of criteria and construct for the creation \\ of a questionnaire on noise exposure
}

\author{
Eduardo Fuentes $\mathrm{L}^{1,2}$, Felipe Cardemil $\mathbf{M}^{1,3}$.
}

\begin{abstract}
RESUMEN
Introducción: La exposición a ruido recreacional y su repercusión sobre la audición es un problema al cual la investigación presta cada vez mayor atención.

Objetivo: Determinar la validez de criterio y constructo de un cuestionario de exposición a ruido recreacional ("CHAR"), así como los factores asociados a ésta.

Material y método: Se realizó un estudio de validación de cuestionario, de corte transversal, en estudiantes universitarios. El protocolo incluyó evaluación audiométrica, consulta de síntomas auditivos relacionados con la exposición a ruido y el cuestionario "CHAR". Se construyeron modelos mediante regresión logística, obteniendo sus respectivas áreas bajo la curva ROC.

Resultados: Se incluyó a 258 estudiantes de la carrera de fonoaudiología. La presencia de escotoma en $4 \mathrm{kHz}$ en el oído derecho obtuvo un área bajo la curva correspondiente a 0,72 (IC 95\%: 0,60-0,84), mientras que en el oído contralateral ésta fue de 0,57 (IC 95\%: 0,49-0,65). El presentar síntomas auditivos luego de asistir a conciertos podría influir en las medidas de validez.

Conclusión: El Cuestionario "CHAR" permite discriminar la presencia de escotoma en la frecuencia $4 \mathrm{kHz}$ del oído derecho. El puntaje de corte va a depender de los objetivos de uso del instrumento; ya sea el orientar en el diagnóstico, o el tamizaje.

Palabras clave: Pérdida auditiva inducida por ruido, aparatos de reproducción musical portátil, curva ROC.
\end{abstract}

1 Programa de Doctorado en Salud Pública, Escuela de Salud Pública, Universidad de Chile.

2 Facultad de Medicina, UDA Ciencias de la Salud, Carrera de Fonoaudiología, Pontificia Universidad Católica de Chile.

3 Servicio de Otorrinolaringología, Hospital Barros Luco Trudeau, Universidad de Chile. 


\section{ABSTRACT}

Introduction: Recreational noise exposure and its impact on hearing is a problem that the investigation is paying more and more attention to.

Aim: To determine the validity of the criteria and construct for the questionnaire on recreational noise exposure (CHAR) and the factors associated.

Materials and Methods: We conducted a validation study for the questionnaire, transversal, using university students. The protocol included audiometric evaluation, consultation on auditory symptoms related to noise exposure and the "CHAR" questionnaire. We constructed it using logistic regression models, obtaining their respective areas under the ROC curve.

Results: It included 258 students in the audiology program. The presence of notch at $4 \mathrm{kHz}$ in the right ear obtained an area under the curve corresponding to $0.72(95 \% \mathrm{Cl}$ : 0.60-0.84) while in the contralateral ear, it was 0.57 (95\% Cl: 0.49-0.65). The auditory symptoms present after attending concerts could influence measures of validity.

Conclusion: The "CHAR" questionnaire allows for discrimination for the presence of notch in the $4 \mathrm{kHz}$ frequency in the right ear. The cutoff score will depend on the objectives for the use of the instrument; to guide the diagnosis or screening.

Key words: Noise induced hearing loss, portable music playback devices, ROC curve.

\section{INTRODUCCIÓN}

La pérdida auditiva inducida por ruido es la segunda causa más común de hipoacusia sensorioneural, superada sólo por la presbiacusia' ${ }^{1}$. Inicialmente el foco de la investigación relacionada al tema estuvo en evaluar el impacto en salud pública de la pérdida auditiva inducida por ruido en contextos ocupacionales; sin embargo, en los últimos años, se ha reportado que varias de las fuentes de ruido que no son de carácter laboral (asistencia a conciertos, eventos o lugares ruidosos, etc.) producen sonidos en los cuales una breve exposición generaría un daño auditivo permanente? ${ }^{2}$.

En relación a la prevalencia del problema, Niskar y cols ${ }^{3}$ estudiaron audiogramas de niños y adolescentes que participaron en la encuesta NHANES III, informando que el 12,5\% (alrededor de 5,2 millones) de los encuestados entre 6 y 19 años presentaron indicios audiométricos sugestivos de pérdida de auditiva inducida por ruido. De manera similar, Rabinowitz y cols ${ }^{4}$ observaron que el $16 \%$ de las audiometrías de adultos jóvenes de entre 17 a 25 años, evidenciaban pérdida auditiva de frecuencias altas. Shargorodsky y cols ${ }^{5}$ al comparar los umbrales audiométricos de adolescentes norteamericanos incluidos en la encuesta NHANES de los años 1988-1994 con los obtenidos entre el año 2005 y 2006 denotaron el aumento, desde el $14,9 \%$ al $19,5 \%$, en la prevalencia de la pérdida auditiva.

En Chile, una de las primeras investigaciones que abordó el estudio de la exposición a ruido recreacional, fue la reportada por Jofré y cols ${ }^{6}$, quienes determinaron que el $30 \%$ de los jóvenes encuestados se encontrarían expuestos a niveles de ruido superiores al límite considerado como riesgoso. La medición se realizó en base al "Cuestionario de exposición a ruido" (CER) ${ }^{7}$. En dicho instrumento, de origen español, se solicita indicar el número de días a la semana de asistencia, horas al día, y el nivel de ruido percibido al asistir a diversas actividades asociadas a la presencia de ruido (escuchar música en mp3, asistir a conciertos, etc.). Con los resultados del cuestionario se realizaron estimaciones de exposición indirectas, trabajando con los valores máximos de nivel continuo equivalente (Leq) reportados en la literatura, lo que podría estar sobreestimando el porcentaje bajo riesgo. Recientemente, Breinbauer y cols ${ }^{8}$ señalaron que la población en riesgo de presentar una pérdida auditiva permanente producto de la utilización de aparatos de reproducción musical portátil podría alcanzar el 12\%. Estos autores nuevamente ponen de manifiesto la relevancia del 
problema, sin embargo, es probable que la magnitud del mismo sea incluso mayor si se consideraran otras fuentes de exposición a ruido recreacional, como la asistencia a discotecas, bares, karaokes 0 eventos deportivos, donde se ha reportado mediante sonometría elevados niveles de ruido ${ }^{9}$.

En cuanto al uso de reportes personales (cuestionarios) para valorar la exposición a ruido recreacional, se ha observado que éstos presentan una alta correlación con las mediciones objetivas realizadas mediante dosimetría ${ }^{10}$. La percepción por parte de los jóvenes que asisten a actividades con ruido recreacional, como conciertos o discotecas, mejoraría a medida que éstos adquieren una mayor experiencia asistiendo a dichos eventos ${ }^{10}$. A su vez, si la valoración acerca de la sonoridad se realiza de forma inmediata, la correlación entre la percepción subjetiva y el LeqA (nivel de presión acústica continuo equivalente ponderado) podría ser aún mayor ${ }^{11}$. En relación al uso de aparatos de reproducción musical, también se ha estudiado el grado de relación que existe entre mediciones subjetivas y objetivas. Se ha constatado que el autorreporte acerca de la intensidad y el tiempo de uso de los aparatos de reproducción musical portátil está fuertemente relacionado con las mediciones objetivas de dichos parámetros ${ }^{12}$.

\section{OBJETIVO}

Considerando lo anterior este trabajo tiene por objetivo determinar la validez de criterio y constructo para la creación del Cuestionario de Hábitos Auditivos ("CHAR") y los factores asociados a ésta. Dicho instrumento permitirá evaluar la exposición a ruido recreacional en jóvenes.

\section{MATERIAL Y MÉTODO}

Se realizó un estudio de corte transversal, de validación de cuestionario, en el período entre agosto de 2011 y diciembre de 2012. Se incluyeron estudiantes de la carrera de fonoaudiología. El protocolo del estudio contó con aprobación del Comité de Ética de la universidad de procedencia de los estudiantes. Cada joven firmó un consentimiento informado para su participación en la investigación.

La universidad de los jóvenes realiza periódicamente programas de pesquisa auditiva en los estudiantes de pregrado de fonoaudiología, siendo este procedimiento considerado como criterio de inclusión al estudio. A su vez, mediante exploración otoscópica y examen audiométrico e impedanciométrico se excluyeron a todos aquellos estudiantes que presentaron patologías de oído no relacionadas a pérdida auditiva inducida por ruido, como por ejemplo otitis media crónica, otopatía fibroadhesiva, otitis media con efusión, otoesclerosis, u otra.

\section{Descripción del cuestionario CHAR}

El "Cuestionario de Hábitos Auditivos Recreacionales" (CHAR) es un instrumento autoadministrado que tiene por objetivo determinar los hábitos auditivos de adolescentes y adultos jóvenes, así como los contextos de exposición a sonidos intensos de carácter recreacional. EI CHAR está compuesto por un apartado acerca de los datos personales del sujeto y 14 preguntas con formato cerrado (de tres a cinco alternativas), en algunos casos con apoyo de imágenes (pregunta 2).

Existe un orden lógico de las preguntas donde es posible reconocer los siguientes aspectos: 1) Características de los reproductores de música personal, periodo, frecuencia y contexto de uso; 2) Asistencia a conciertos; y 3) Asistencia a otros recintos ruidosos. Cada pregunta tiene un puntaje máximo de 5 puntos, asignándolo en forma creciente en relación al nivel de ruido asociado a cada conducta o a la frecuencia de ésta. Así por ejemplo, en la pregunta 4 , en la que el sujeto es consultado acerca del volumen en que utiliza el reproductor de música portátil, se otorga un punto en el caso de indicar "Muy bajo (cercano al mínimo disponible)" y cinco puntos en el caso de señalar la opción "Muy alto (cercano al máximo disponible)".

En cuanto a los antecedentes, además del sexo y la edad se consideró consultar por la existencia de antecedentes familiares de hipoacusia. Se consideró como variable el sexo de los estudiantes ya que se ha reportado que la susceptibilidad al ruido puede ser influenciada por éste ${ }^{13,14}$. A su vez, la edad es un factor importante ya que la exposición a ruido recreacional anual decrece con la edad ${ }^{15}$. Finalmente, consultar por antecedentes familiares de hipoacusia se fundamentó en el hecho que presentar dicho historial ha sido asociado a una mayor susceptibilidad a manifestar pérdida auditiva inducida por ruido ${ }^{16,17}$. 
Aplicaciones previas del instrumento han permitido constatar, mediante análisis exploratorio factorial, la existencia de los tres ítemes mencionados anteriormente. Además, la consistencia interna del instrumento, evaluada a través del Coeficiente Alpha de Cronbach lanzó un valor de 0,81 para el cuestionario en su totalidad ${ }^{18}$.

\section{Validación de criterio a través de los resultados audiométricos}

El cuestionario CHAR fue aplicado a una muestra intencionada de jóvenes universitarios, a los que también se les realizó una evaluación audiométrica. Esta última tuvo por objetivo determinar la existencia de una asociación entre el puntaje obtenido en el cuestionario y la presencia de un escotoma en las frecuencias agudas. La audiometría se efectuó en cabina silente (sonoaislada y sonoamortiguada) con un audiómetro Interacoustics AC40 calibrado según norma ANSI S3.6. Los umbrales tonales se midieron en las frecuencias de 0,25 a $8 \mathrm{kHz}$, incluyendo interoctavas (frecuencias de 3 y $6 \mathrm{kHz}$.), de acuerdo con la norma ISO 8253-1, utilizando una modificación del procedimiento de HughsonWestlake ${ }^{19}$. Dado que el audiómetro contaba con auriculares TDH-39 se realizó un corrección de 6 $\mathrm{dB}$ en el umbral obtenido en la frecuencia $6 \mathrm{kHz}$ para evitar la presencia artificial de escotoma ${ }^{20}$. Se determinó la presencia de escotoma de acuerdo al criterio propuesto por Coles y cols ${ }^{21}$, en donde el nivel del umbral de audición en 3 y/o 4 y/o 6 $\mathrm{kHz}$ debe ser al menos $10 \mathrm{~dB}$ superior a 1 ó 2 y $8 \mathrm{kHz}$. Finalmente, con el objetivo de excluir los cambios transitorios de umbral sólo se realizaron los exámenes audiométricos a aquellos estudiantes que indicaron no exponerse a ruido las últimas 24 horas previas (uso de reproductores de archivos $\mathrm{mp3}$, asistencia a conciertos, etc.).

\section{Validación de constructo a través de los síntomas auditivos percibidos}

Adicionalmente, los estudiantes completaron el "Cuestionario de salud auditiva" el que se basó en The hearing symptom description scale (HSD) ${ }^{22}$. Dicho instrumento constó de siete preguntas de formato cerrado, con respuesta en su mayoría del tipo SI/NO, siendo suministrado a modo de entrevista. La aplicación tuvo por objetivo evaluar la autopercepción de síntomas tales como: tinnitus temporal/permanente, pérdida auditiva, dolor después de la exposición a sonidos intensos, etc.

\section{Análisis estadístico}

Para el análisis estadístico de los datos obtenidos en la presente investigación se utilizó el programa STATA versión 12. Las variables cuantitativas continuas son descritas mediante promedio y desviación estándar (DE), o mediana, rango intercuartílico (RIC), y valores mínimo y máximo, según correspondiera. En el caso de la variables categóricas se reportaron las frecuencias absolutas y relativas. Se constató a través de la prueba de Shapiro-Wilk que la variable "umbrales audiométricos" no poseía una distribución normal, por lo que se utilizó el test no paramétrico de Friedman para comparar las frecuencias evaluadas. Una vez categorizada la anterior variable, en base a la definición de escotoma propuesta por Coles y cols ${ }^{21}$, se procedió a crear modelos estadísticos a través de regresión logística. Cada modelo tuvo como variable desenlace la presencia de alteraciones en los umbrales auditivos en la forma de escotoma en las frecuencias agudas 3,4 y $6 \mathrm{kHz}$ y como variable predictora el puntaje obtenido en el "CHAR". Se obtuvo el área bajo la curva ROC (Receiver Operator Characteristic) para cada modelo propuesto y se determinó la sensibilidad y especificidad para los posibles puntos de corte del puntaje obtenido en el cuestionario "CHAR". Adicionalmente, se reportó el índice de Youden (J), ya que éste permite combinar sensibilidad y especificidad en un solo indicador; asignándole un mismo peso a ambas medidas ${ }^{23}$. Finalmente, se evaluó a través de regresión logística la asociación entre la autopercepción de síntomas auditivos (tinnitus, hipoacusia, etc.) y el puntaje obtenido en el "CHAR". Se consideró un nivel de significancia del $5 \%$ para todos los contrastes de hipótesis realizados.

\section{RESULTADOS}

\section{Validez de criterio utilizando audiometría}

La muestra la constituyeron 259 estudiantes universitarios. La edad promedio correspondió a 21,2 años $( \pm 2,11)$ con un mínimo de 18 años y un 
máximo de 30 . En cuanto al sexo, el $73,46 \%$ de los sujetos fueron mujeres y el $23,57 \%$ hombres. A su vez, el 21,4\% de los sujetos indicó presentar antecedentes familiares de hipoacusia $(n=55)$.

En cuanto a los umbrales audiométricos, las medianas en todas las frecuencias no superaron el límite de $20 \mathrm{~dB}$ considerado como normal. Sin embargo, existen diferencias significativas entre las frecuencias agudas evaluadas (2 kHz-8 kHz), tanto en el oído izquierdo ( $p$ $<0,001)$ como en el derecho $(p<0,001)$. Además, se observaron algunos valores por sobre los $20 \mathrm{~dB}$ los que fueron considerados "outliers"; la frecuencia $6 \mathrm{kHz}$ en ambos oídos fue la que evidenció la mayor elevación del umbral audiométrico (Figuras 1 y 2).

Tanto el modelo cuya respuesta fue la presencia de escotoma en $4 \mathrm{kHz}$ del oído izquierdo como en $6 \mathrm{kHz}$ del derecho, no resultaron estar asociados al puntaje obtenido en el cuestionario CHAR, por lo que no se estimó su correspondiente área bajo la curva ROC. En el caso del modelo cuya variable respuesta fue la pre- sencia de escotoma en $6 \mathrm{kHz}$ en el oído izquierdo, su área bajo la curva alcanzó un valor de 0,57 (IC 95\%: $0,49-0,65)$. Para el modelo cuya variable respuesta fue la presencia de escotoma en $4 \mathrm{kHz}$ en el oído derecho, su área bajo la curva correspondió a 0,72 (IC 95\%: 0,60-0,84) (Figura 3). Dada su mayor área bajo la curva ROC se decide considerar los resultados de este último modelo, para establecer el punto de corte del Cuestionario de Hábitos Auditivos Recreacionales "CHAR". Para cada punto de corte se obtiene tanto sensibilidad como especificidad (Tabla 1).

Al observar la tabla es posible mencionar que la sensibilidad aumenta rápidamente a medida que se disminuye el puntaje de corte del cuestionario CHAR, lo inverso ocurre con la sensibilidad. Tentativamente es posible fijar el corte en 35-36 puntos, dado que se obtiene un equilibrio entre sensibilidad y especificidad. Considerando los anteriores puntos de corte el porcentaje de sujetos correctamente clasificados podría variar entre $63,4 \%$ y $63,7 \%$.
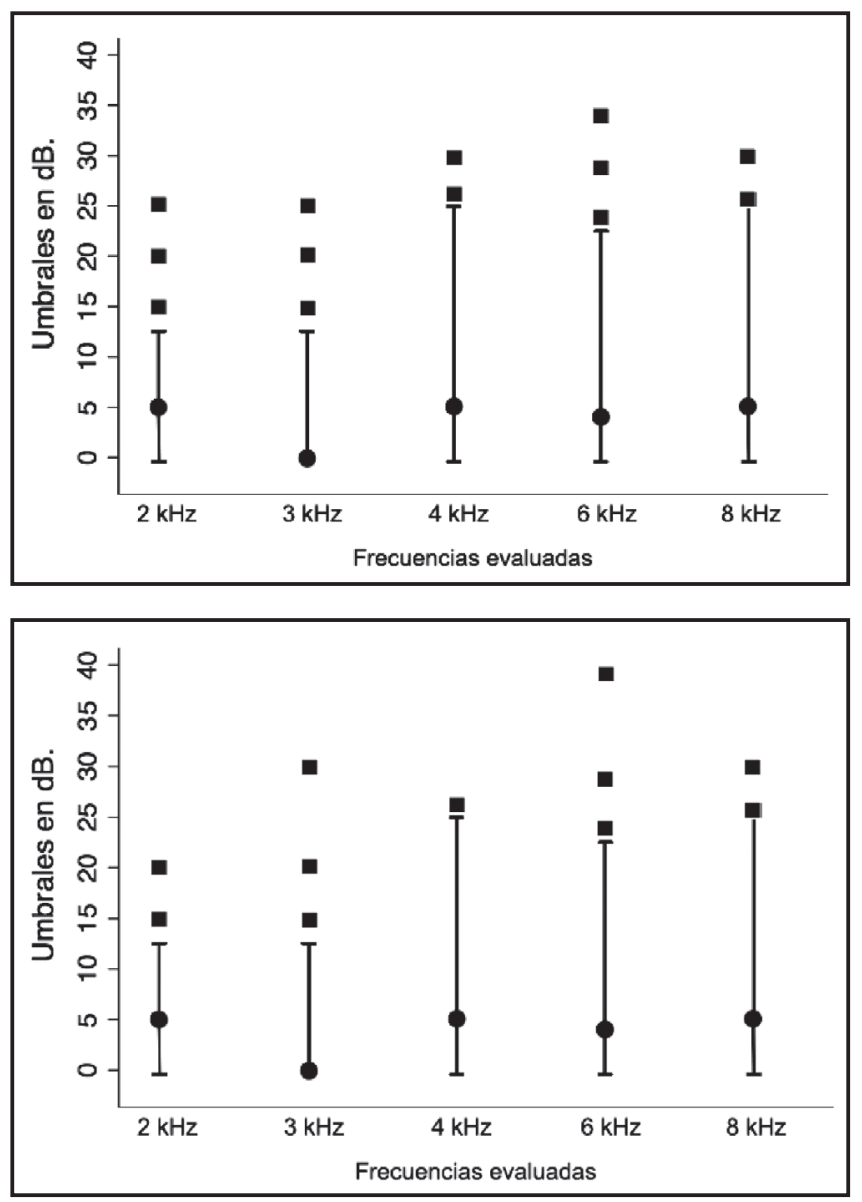

Figura 1. Cuartiles superior e inferior de los umbrales audiométricos del oído derecho. Los círculos corresponden a las medianas y los cuadrados a los "outliers".

Figura 2. Cuartiles superior e inferior de los umbrales audiométricos del oído izquierdo. Los círculos corresponden a las medianas y los cuadrados a los "outliers". 
Figura 3. Área bajo la curva para la variable respuesta escotoma en $4 \mathrm{kHz}$ del oído derecho.

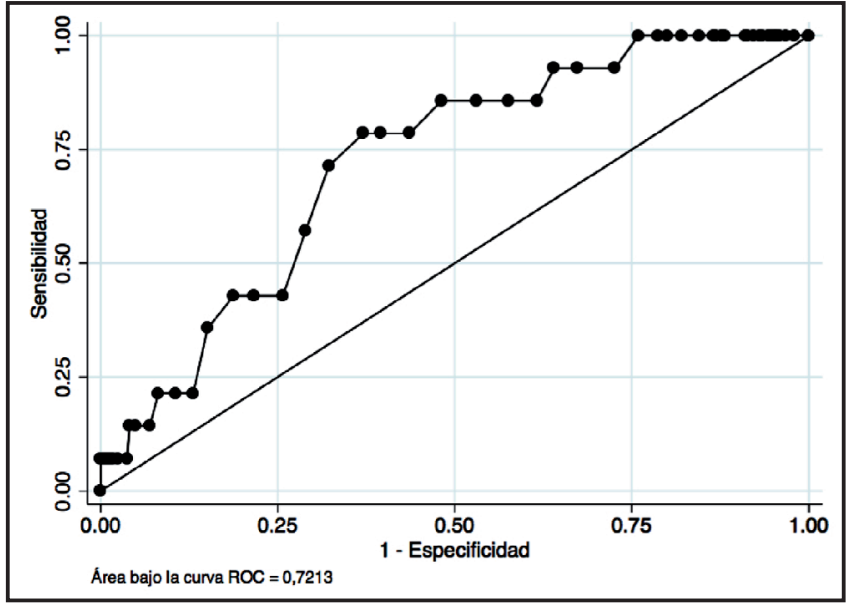

Tabla 1. Punto de corte y respectivo porcentaje de sensibilidad y especificidad para el cuestionario de hábitos auditivos recreacionales

\begin{tabular}{|lccc|}
\hline Puntaje en el CHAR & Sensibilidad (\%) & Especificidad (\%) & Correctamente clasificados (\%) \\
\hline Mayor o igual a 28 & 92,86 & 32,65 & 35,91 \\
Mayor 0 igual a 29 & 92,86 & 35,92 & 39,00 \\
Mayor o igual a 30 & 85,71 & 38,37 & 40,93 \\
Mayor 0 igual a 31 & 85,71 & 42,45 & 44,79 \\
Mayor o igual a 32 & 85,71 & 46,94 & 49,03 \\
Mayor o igual a 33 & 85,71 & 51,84 & 53,67 \\
Mayor o igual a 34 & 78,57 & 56,33 & 57,53 \\
Mayor o igual a 35 & 78,57 & 60,41 & 61,39 \\
Mayor o igual a 36 & 78,57 & 62,86 & 63,71 \\
Mayor 0 igual a 37 & 71,43 & 67,76 & 67,95 \\
\hline
\end{tabular}

El índice de Youden (J) en este caso correspondió a 0,41. Según esto, con un puntaje igual o mayor a 36 puntos en el CHAR se obtiene el equilibrio entre sensibilidad y especificidad $(78,57 \%$ y $62,86 \%$, respectivamente). Dicho punto de corte concuerda con los valores reportados en la Tabla 1. A su vez, si se busca mejorar la sensibilidad, el puntaje de corte en 29 parece ser una buena alternativa. Teniendo en cuenta los valores de prevalencia del $12,9 \%$ para la pérdida auditiva inducida por ruido en jóvenes reportados por Henderson y cols ${ }^{24}$ el valor predictivo positivo alcanzó $57,93 \%$ y el valor predictivo negativo $42,78 \%$. Aunque es común que los valores predictivos (sobre todo los positivos) varíen según la prevalencia, en este caso se mantienen relativamente estables (Tabla 2).

Si bien el cuestionario "CHAR" es un instrumento que incluyó preguntas relacionadas con la exposición a ruido recreacional, una de las 14 preguntas (pregunta 12) hizo referencia a la presencia de síntomas auditivos luego de la asistencia a conciertos. Dentro de los síntomas que se listaron se encontraban: subir el volumen del teléfono para escuchar mejor, solicitar la repetición de palabras no oídas y el escuchar sonidos o pitos en los oídos. Considerando en el modelo a aquellos sujetos que no han experimentado ningún síntoma ( $n=146$ ), el área bajo la curva ROC alcanzó el valor de 0,77 (IC 95\%: 0,56-0,98). El punto donde

Tabla 2. Valor predictivo positivo y negativo según prevalencia de pérdida auditiva inducida por ruido

\begin{tabular}{|ccc|}
\hline $\begin{array}{c}\text { Prevalencia } \\
(\%)\end{array}$ & $\begin{array}{c}\text { Valor predictivo } \\
\text { positivo }\end{array}$ & $\begin{array}{c}\text { Valor predictivo } \\
\text { negativo }\end{array}$ \\
\hline 12 & 58,08 & 42,62 \\
14 & 57,77 & 42,94 \\
16 & 57,54 & 43,17 \\
18 & 57,35 & 43,35 \\
20 & 57,21 & 43,50 \\
25 & 56,95 & 43,75 \\
30 & 56,78 & 43,92 \\
\hline
\end{tabular}


se obtuvo valores semejantes de sensibilidad y especificidad se ubicó en 33 puntos con $80 \%$ y $73,1 \%$, respectivamente. El porcentaje de sujetos clasificados correctamente alcanzó el $73,3 \%$. El punto donde se obtuvo la mayor sensibilidad fue 26 puntos, alcanzando el $100 \%$, en desmedro de la especificidad que llegó al 35,5\%. Algo diferente ocurre para aquellos que habían presentado al menos uno de los síntomas listados anteriormente $(n=113)$, donde el área bajo la curva fue 0,62 (IC 95\%: 0,43-0,82). El punto de balance entre sensibilidad y especificidad se alcanzó a los 37 puntos con $88,89 \%$ y $39,42 \%$, respectivamente. El porcentaje de sujetos clasificados correctamente fue el $43,36 \%$. El punto donde se obtuvo la mayor sensibilidad fue 29 puntos, alcanzando el $100 \%$, en desmedro de la sensibilidad que llegó al 13,46\%.

Con el propósito de determinar la influencia de las variables sexo, antecedentes familiares de hipoacusia y edad, las que fueron consideradas por el cuestionario CHAR pero no cuantificadas y con esto incluidas en el puntaje final, se procedió a generar modelos mediante regresión logística, calculando su área bajo la curva ROC. Se consideró como referencia a comparar el modelo donde el único predictor fue el puntaje obtenido en el cuestionario CHAR y la variable respuesta la presencia de escotoma en la frecuencia $4 \mathrm{kHz}$ del oído derecho. En cuanto al sexo éste no constituyó un predictor significativo (OR: 1,04; IC 95\%: 0,30-3,60), y como era de esperar la diferencia de áreas bajo la curva ROC en relación al modelo de referencia tampoco fue significativa $(p=0,91)$. Lo mismo puede ser dicho sobre la variable "edad", donde el área bajo la curva ROC no sufrió cambios significativos ( $p=0,37$ ). En el caso de la variable "presencia de antecedentes de hipoacusia", resultó ser un predictor significativo; la posibilidad de presentar escotoma en la frecuencia $4 \mathrm{kHz}$ del oído derecho aumenta 4,06 veces (IC 95\%: 1,24-13,31) al estudiante indicar antecedentes familiares de pérdida auditiva. Sin embargo, al comparar las áreas bajo la curva ROC con la obtenida para el modelo de referencia, ésta no cambió significativamente $(p=0,35)$ (Figura 4).

\section{Validez de constructo utilizando el autorreporte de síntomas auditivos}

En cuanto a los resultados del "Cuestionario de Salud Auditiva", el puntaje obtenido en el cuestionario "CHAR" resultó ser un predictor significativo tanto para tinnitus que se prolongó más de 24 horas y el experimentar tinnitus después de la exposición a ruido (Tabla 3). Por cada punto más en el cuestionario CHAR, la posibilidad de presentar tinnitus que se prolongó más de 24 horas aumentó 1,09 veces (IC 95\%: 1,03-1,15). En este caso el área bajo la curva ROC alcanzó un valor de 0,69 (IC 95\%: 0,59-0,80). A su vez, por cada punto más en el cuestionario CHAR, la posibilidad haber experimentado pitidos o zumbidos en la cabeza o los oídos después de la exposición a ruido aumentó 1,04 veces (IC 95\%: 1,02-1,07). Para este último modelo el área bajo la curva ROC alcanzó un valor de 0,62 (IC 95\%: 0,55-0,70).

\section{DISCUSIÓN}

Se realizó una validación de criterio del cuestionario CHAR en una muestra de 259 estudiantes universitarios chilenos. El propósito del instrumento fue determinar

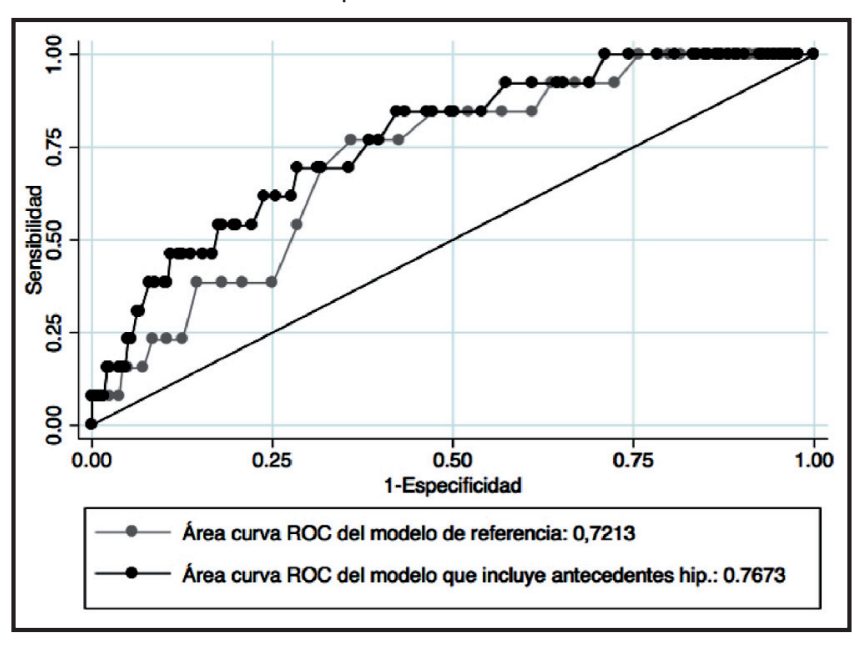

Figura 4. Área bajo la curva del modelo de referencia (puntaje en el CHAR como predictor) y el que incluye además la presencia de alteraciones auditivas a nivel familiar. 
Tabla 3. Asociación entre la autopercepción de síntomas auditivos y el puntaje del Cuestionario de Hábitos Auditivos

\begin{tabular}{|lcc|}
\hline Pregunta & OR & IC 95\% \\
\hline 1. ¿Considera que presenta algún problema para escuchar? & 0,99 & $0,96-1,02$ \\
2. ¿Alguna vez ha tenido zumbidos o pitidos en los oídos que se prolongaron durante más de 24 horas? & 1,09 & $1,03-1,15^{*}$ \\
3. ¿Percibe zumbidos o pitidos en la cabeza o los oídos de forma permanente? & 1,02 & $0,97-1,06$ \\
4. ¿Alguna vez ha experimentado dolor en los oídos después de la exposición a un ruido? & 0,99 & $0,97-1,02$ \\
5. ¿Alguna vez ha experimentado pitidos o zumbidos en la cabeza o los oídos después de la exposición a ruido? & 1,04 & $1,02-1,07^{*}$ \\
6. ¿Alguna vez ha experimentado dificultades para escuchar después de la exposición a ruido? & 1,01 & $0,99-1,03$ \\
7. ¿Siente que es más sensible al ruido que otras personas? & 0,99 & $0,97-1,02$ \\
\hline
\end{tabular}

OR: Odds ratio. * Significativo, $p<0,01$.

Ios hábitos auditivos de jóvenes estudiantes, y con esto el nivel de exposición a ruido recreacional.

En cuanto al proceso de validación se realizó a través del contraste con un criterio externo (Gold standard) como lo es la audiometría. La evaluación consideró interoctavas como 3 y $6 \mathrm{kHz}$ dado que son éstas las que junto con $4 \mathrm{kHz}$ se ven comprometidas tempranamente al existir una pérdida auditiva inducida por ruido ${ }^{20}$. Además, los umbrales auditivos obtenidos fueron corregidos, restando $6 \mathrm{~dB}$, considerando que el audiómetro contaba con auriculares que artificialmente podrían elevarlos (TDH-39) ${ }^{21,25}$.

En relación a las diferencias entre las distintas frecuencias evaluadas por oído, éstas fueron significativas, destacando la presencia de sujetos con elevación del umbral por sobre lo normal $(20 \mathrm{~dB})$ en las frecuencias 4 y $6 \mathrm{kHz}$. Esta última, a pesar de la corrección realizada, mostró las mayores desviaciones individuales. En cuanto a la significancia de este hallazgo, dado que la sensibilidad auditiva es variable en diferentes frecuencias, es posible que un audiograma de un sujeto normal pueda presentar un escotoma en $6 \mathrm{kHz}$, si el estándar de referencia para dicha frecuencia es demasiado bajo26. Autores como Osei-Lah y Yeoh observaron una gran cantidad de escotomas en la frecuencia $6 \mathrm{kHz}(45,3 \%)$, esto a pesar de aplicar una corrección como en la del presente estudio, sin embargo, se inclinaron por asumir que éstos fueron artificialmente creados ya que un gran porcentaje de la muestra $(50 \%)$ no presentaba ningún antecedente médico relevante ni historial de exposición a ruido ${ }^{20}$. Algo similar podría estar ocurriendo en la presente investigación, ya que si bien existe una asociación entre el puntaje en el cuestionario CHAR y la presencia de escotoma en la frecuencia 6 kHz, el área bajo la curva ROC es pobre.

En cuanto al área bajo la curva ROC del modelo cuya variable respuesta fue la presencia de escotoma en la frecuencia $4 \mathrm{kHz}$, ésta podría ser considerada aceptable ${ }^{27}$. Dichos hallazgos son concordantes a los reportados por Kim y cols $^{28}$ quienes observaron la elevación del umbral en el mismo oído y frecuencia en aquellos usuarios de reproductores de música portátil de larga data (más de 5 años continuos 015 años acumulados). En este caso es posible señalar que existe una asociación entre la exposición a ruido recreacional y la presencia de escotoma en la frecuencia $4 \mathrm{kHz}$ del oído derecho, la cual podría ser discriminada por el puntaje obtenido en el cuestionario "CHAR". Es necesario recordar que en cuanto al puntaje de corte, depende de los fines por los que fue creado el instrumento. En algunos casos, al tratarse de un test de apoyo al diagnóstico se buscará maximizar tanto sensibilidad como especificidad, por lo que fijar el corte en 36 puntos sería lo indicado. En otros escenarios se buscará obtener la mayor sensibilidad, como lo es en el caso de los test de tamizaje/screening, para los que un punto de corte en 29 puntos parece ser adecuado.

En relación a los factores que resultaron estar asociadas a escotoma en $4 \mathrm{kHz}$, destacó la presencia de antecedentes familiares de hipoacusia, sin embargo el área bajo la curva ROC no sufrió cambios significativos, por lo que no se justifica la generación de puntajes de corte diferenciados según dicha variable.

En cuanto a la asociación de los síntomas auditivos referidos por los estudiantes, algunos de éstos fueron asociados al puntaje obtenido en el cuestionario CHAR (relacionados con tinnitus). A su vez, el presentar síntomas auditivos luego de asistir 
a conciertos influiría en las medidas de validez del cuestionario CHAR, aumentando la sensibilidad y confiabilidad de éste, en aquellos sujetos que no reportaron presentar los mencionados síntomas. Es posible que el haber experimentado síntomas auditivos luego de la exposición a ruido al asistir a conciertos haga cambiar las conductas auditivas de los sujetos y con esto las respuestas entregadas en el cuestionario no reflejen fielmente sus historiales de exposición. Beach y cols ${ }^{29}$ constataron que dentro de las claves para adoptar medidas de protección cuando por ejemplo se asiste a conciertos, estaba la presencia de tinnitus, experimentar una pérdida temporal de la audición u otros signos de daño al sistema auditivo. Otros estudios también apoyan la idea de que experimentar síntomas auditivos (tinnitus) se asocia con las actitudes negativas hacia el ruido y a escuchar música a altos niveles, a su vez, estas últimas se relacionan a un mayor uso de protección auditiva al asistir a conciertos por parte de los adolescentes ${ }^{30}$. Considerando Io anterior sería aconsejable diferenciar entre aquellos estudiantes que han 0 no experimentado síntomas auditivos, al momento de seleccionar el puntaje de corte del cuestionario "CHAR".

Estos resultados podrían fundamentar considerar el cuestionario CHAR como una herramienta útil en la pesquisa temprana de aquellos sujetos con conductas auditivas riesgosas para presentar una pérdida auditiva inducida por ruido. Todo esto con el propósito de disminuir la exposición temprana a ruido ya que se ha reportado que aquellos oídos expuestos a temprana edad no "envejecen" en la misma medida que los no expuestos, presentando un ritmo mayor en cuanto al descenso en los umbrales audiométricos ${ }^{31-34}$.

\section{CONCLUSIONES}

El Cuestionario de Hábitos Auditivos Recreacionales (“CHAR") permite discriminar la presencia de escotoma en la frecuencia $4 \mathrm{kHz}$ del oído derecho. El área bajo la curva ROC es considerada aceptable; alcanzando una sensibilidad del $78,57 \%$ y una especificidad del $62,86 \%$. El puntaje de corte va a depender de los objetivos de uso del presente instrumento; si éste es la pesquisa temprana se recomienda fijar el punto de corte en 29 puntos. El presentar síntomas auditivos luego de asistir a conciertos influiría en las medidas de validez del cuestionario CHAR; existiendo un aumento de sensibilidad y especificidad para el caso de los sujetos que aún no experimentan dichos síntomas.

\section{BIBLIOGRAFÍA}

1. Rabinowitz PM. Noise-induced hearing loss. Am Fam Physician 2000; 61: 2749-56, 2759-60.

2. Rabinowitz PM. The Public Health Significance of Noise-Induced Hearing Loss. En: Le Prell G y cols, eds, Noise-Induced Hearing Loss: Scientific Advances. New York: Springer 2012; 13-25.

3. Niskar AS, Kieszak SM, Holmes AE, Esteban E, Rubin C, Brody DJ. Estimated Prevalence of NoiseInduced Hearing Threshold Shifts Among Children 6 to 19 Years of Age: The Third National Health and Nutrition Examination Survey, 1988-1994, United States. Pediatrics 2001; 108: 40-3.

4. Rabinowitz, PM, Slade MD, Galusha D, Dixon-Ernst C, Cullen MR. Trends in the prevalence of hearing loss among young adults entering an industrial workforce 1985 to 2004. Ear \& Hearing 2006; 27: 369-75.

5. Shargorodsky J, Curhan SG, Curhan GC, Eavey $R$. Change in prevalence of hearing loss in US adolescents. JAMA 2010; 304: 772-8.

6. Jofré D, De la Paz F, Platzer L, Anabalón JL, Grasset E, Barnafin N. Evaluación de la exposición a ruido social en jóvenes chilenos. Rev Otorrinolaringol Cir Cabeza Cuello 2009; 69: 23-8.

7. Zenker F, Altahona MP, Barajas JJ. La exposición a ruido por actividades de ocio en adolescentes. Rev Logop Fon Adiol 2001; 21: 173-80.

8. Breinbauer ha, Anabalon Jl, Gutiérrez D, Caro J. Estimación de riesgos y hábitos de uso de reproductores de música personal en una muestra de población chilena. Rev Otorrinolaringol Cir Cabeza Cuello 2011; 71: 31-8.

9. Beach E, Willams W, Gilliver M. Estimating Young Australian Adults' Risk of Hearing Damage From Selected Leisure Activities. Ear \& Hearing 2013; 34: 75-82.

10. Beach E, Williams W, Gilliver M. The objectivesubjective assessment of noise: Young adults can estimate loudness of events and life style noise. International Journal of Audiology 2012; 51: 444-9.

11. Kuwano S, Namba S, Kato T. Auditory memory and evaluation of environmental sounds. Proceedings of Acoustics 08. Paris, 2008: 1171-4. 
12. Portnuff CD, Fligor BJ, Arehart KH. Self-report and long-term fi eld measures of MP3 player use: How accurate is self-report? International Journal of Audiology 2013; 52: 33-40.

13. Berger EH, Royster LH, Thomas WG. Presumed noise-induced permanent threshold shift resulting from exposure to an A-weighted Leq of $89 \mathrm{~dB}$. Journal of the Acoustical Society of America 1978; 64: 192-7.

14. Niskar AS, Kieszak SM, Holmes Ae, Esteban E, Rubin C, Brody DJ. Estimated Prevalence of NoiseInduced Hearing Threshold Shifts Among Children 6 to 19 Years of Age: The Third National Health and Nutrition Examination Survey, 1988-1994, United States. Pediatrics 2001; 108: 40-3.

15. Beach E, Williams W, Gilliver M. Leisure noise exposure: Participation trends, symptoms of hearing damage, and perception of risk. International Journal of Audiology 2013; 52: 20-5.

16. McMahon CM, Kifley A, Rochtchina E, Newall P, Mitchell P. The contribution of family history to hearing loss in an older population. Ear Hear 2008; 29: 578-84.

17. Abreu-Silva RS, Rincon D, Horimoto AR, Sguillar AP, Ricardo LA, Kimura L, Batissoco AC, Auricchio MT, Otto PA, Mingroni-Netto RC. The search of a genetic basis for noise-induced hearing loss (NIHL). Ann Hum Biol 2011; 38: 210-8.

18. Fuentes E. Hábitos Auditivos Recreacionales en Estudiantes Universitarios. XXX Jornadas Chilenas de Salud Pública; Escuela de Salud Pública de la Universidad de Chile. 8 - 9 Noviembre 2012.

19. International Organization of Standardization. Acoustics. Audiometric test methods-Part 1: Basic pure tone air and bone conduction threshold audiometry. Geneva: ISO. 1989.

20. OseI-LAH V, YeOH LH. High frequency audiometric notch: An outpatient clinic survey. International Journal of Audiology 2010; 49: 95-8.

21. Coles R, Lutman ME, Buffin J. Guidelines on the diagnosis of noise-induced hearing loss for medicolegal purposes. Clin Otolaryngol 2000; 25: 264-73.

22. Olsen-Widén SE, ErLAndsson SI. The influence of socio-economic status on adolescent attitude to social noise and hearing protection. Noise and Health 2004; 25: 59-70.

23. Szkıo M, Nieto F. Epidemiology: Beyond the Basics. 2a ed. Boston: Jones and Bartlett Publishers 2013; 516.

24. Henderson E, Testa Ma, Hartnick H. Prevalence of Noise-Induced Hearing-Threshold Shifts and Hearing Loss Among. Pediatrics 2011; 127: 39-46.

25. LutMAn ME, QASEM HY. A source of audiometric notches at 6 kHz. En: Prasher D, Luxon LM, eds, Biological Effects of Noise. London: Wiley, 2006; 170-6.

26. RoBinson DW. Threshold of hearing as a function of age and sex for the typical unscreened population. Br J Audiol 1988; 22: 5-20.

27. Streiner DL, Cairney J. What's under the ROC? An introduction to receiver operating characteristics curves. Canadian Journal of Psychiatry 2007; 52: 121-8.

28. KIm MG, Hong SM, Shim HJ, KIm YD, Cha Cl, YeO $S G$. Hearing threshold of Korean adolescents associated with the use of personal music players. Yonsei Med J 2009; 50: 771-6.

29. Beach EF, Williams W, Gilliver M. A qualitative study of earplug use as a health behavior: The role of noise injury symptoms, self-efficacy, and an affinity for music. Journal of Health Psychology 2012; 17: 237-46.

30. WIDÉN SE, Holmes AE, ERLANDSSON SI. Reported hearing protection use in young adults from Sweden and the USA: Effects of attitude and gender. International Journal of Audiology 2006; 45: 273-80.

31. Gates GA, Schmid P, Kujawa SG, Nam B, D'Agostino R. Longitudinal threshold changes in older men with audiometric notches. Hear Res 2000; 141: 220-8.

32. Rosenhal $U$. The influence of ageing on noiseinduced hearing loss. Noise and Health 2003; 5: 47-53.

33. SCHMIEDT RA. The Physiology of Cochlear Presbycusis. En: Gordon-Salant Sy cols, eds, The Aging Auditory System. New York: Springer, 2010; 9-38.

34. KujaWa SG, Liberman MC. Acceleration of AgeRelated Hearing Loss by Early Noise Exposure: Evidence of a Misspent Youth. The Journal of Neuroscience 2006; 26: 2115-23.

\author{
Dirección: Eduardo Fuentes L. \\ Escuela de Fonoaudiología, Pontificia Universidad Católica de Chile. \\ Avenida Vicuña Mackenna 4860, Macul, Santiago. \\ E mail: eduardo.fuentes@uc.cl
}

